\title{
Nikotinamid İçeren Çinko (II) Kompleksinin Moleküler Özellikleri ve Titreşim Spektrumu
}

\author{
Molecular Properties and Vibrational Spectra of Zinc (II) Complex with Nicotinamide
}

\author{
Hacer GÜMÜŞ*
}

Kocaeli Üniversitesi, Gölcük MYO, 41380, Kocaeli

\author{
• Geliş tarihi / Received: 01.02.2018 • • Düzeltilerek geliş tarihi / Received in revised form: 12.08.2018 • Kabul tarihi / Accepted: 21.08 .2018
}

\section{Öz}

Bu makalede, $\left(\mathrm{C}_{6} \mathrm{H}_{11} \mathrm{~N}_{2}\right)\left[\mathrm{ZnBr}_{3}\left(\mathrm{C}_{6} \mathrm{H}_{6} \mathrm{~N}_{2} \mathrm{O}\right)\right]$ (1a) molekülünün yapısal parametreleri üzerine teorik bir çalışma rapor edilmiştir. Molekül 1a için moleküler geometrik yapısı, harmonik titreşim dalgasayıları, moleküler sınır orbital (HOMO-LUMO) enerjileri, Mulliken, APT ve NBO atom yükleri ve moleküler elektrostatik potansiyel (MEP) yüzeyleri Gaussian programı kullanılarak hesaplanmıştır. Gaussian programında hesaplanan teorik hesaplamaların tümü DFT/B3LYP ve DFT/HSEH1PBE metotları ile 6-311++G(d,p) seti ile hesaplanmıştır.

Anahtar kelimeler: DFT, Dipol moment, IR ve Raman spektroskopi, Moleküler orbital enerji

\begin{abstract}
In this paper, we report a theoretical study on structure parameters of $\left(\mathrm{C}_{6} \mathrm{H}_{11} \mathrm{~N}_{2}\right)\left[\mathrm{ZnBr}_{3}\left(\mathrm{C}_{6} \mathrm{H}_{6} \mathrm{~N}_{2} \mathrm{O}\right)\right]$ (la) molecule. Molecular geometry, harmonic vibration waves, molecular frontier orbital (HOMO-LUMO) energies, mulliken, APT and NBO atomic charges and molecular electrostatic potential (MEP) surfaces were calculated for molecule la by using the Gaussian program. All of the theoretical calculations calculated in the Gaussian program were calculated using B3LYP and HSEHIPBE methods at 6-311 ++ G(d,p) set.
\end{abstract}

Keywords: DFT, Dipole moment, IR and Raman spectroscopy, Molecular orbital energy

\footnotetext{
* Hacer GÜMÜŞ; hacer.gumus@kocaeli.edu.tr; Tel: (0262) 42238 55; orcid.org/0000-0002-6486-0515
} 


\section{Giriş}

NIA olarak kısaltılmış olan Nikotinamid B vitamini kompleksinin bir bileşeni olarak bilinmesinin yanı sıra koenzimlerin nikotinamid adeninin önemli bir bileşeni olduğu kadar dinükleotid (NAD) ve nikotinamid adenin dinükleotidi fosfat (NADP) gibi birçok biyolojik oksidasyon - redüksiyon reaksiyonlarını içerir (Bolukbasi ve Akyuz, 2005). Bir piridin türevi olan Nikotinamid, insan organizmasının metabolik süreçlerinde ortaya çıkan önemli bir biyoligantdır. Genellikle bitkilerde ve insan dokularında (Shibata, 1994; Sismanoglu, 2003) baskındır ve bir koenzim ile önemli biyolojik aktiviteyi gösterir (Akalin ve Akyuz, 2006) ve nikotinamide adenin dinükleotid ve nikotinamid adenin dinükleotid fosfat iyonları olarak geniş ilgi alanlarına sahiptir (Magel vd., 2001). Nikotinamid, iltihap önleyici özelliği nedeniyle, ilaç endüstrisinde yaşamsal bir bileşik olmasının farmakolojik önemini de ortaya koymaktadır (Raj vd., 2000; Ide vd., 2002). Bir diğer taraftan, çinko metal kompleks sentezinde kritik bir rol oynamaktadır ve ağn krizinin önlenmesi ve çeşitli hastalıkların tedavisi ile anti-bakteriyel ve antiviral etkinlikler açısından tedavi edici ajanlardır (Pasaoglu vd., 2006). Ayrica, Piridinkarboksamidin izomeri olan nikotinamid demir kaynakl1 böbrek hasarlarını azaltmayı içeren aktiviteye sahip tıbbi ajanlardan oluşan bir sınıftır (Bakiler vd., 2007).

\section{Bulgular ve Tartışma}

\subsection{Kristal Yapıst}

Molekül 1a'nın kristal yapısı Chunyan vd. (2015) tarafindan sentezlenmiştir. Sentezlenen bu molekülün X-ışını tek kristal yapısı CCDC 994368 kodu ile Cambridge kristallografik yap1 veri tabanında (CSD) bulunmaktadır. Molekül 1a'nın moleküler ağırlığı 538,39 $\mathrm{g} \mathrm{mol}^{-1}$, birim hücresi monoklinik yapıda ve $P 2(1) / c$ uzay grubundandır. Molekül la'nın deneysel yapısı ve atom numaraları Şekil 1'de gösterilmiştir (Chunyan vd., 2015).

\subsection{Geometrik Optimizasyonu}

Molekül 1a'nın teorik olarak geometrik yapısını incelemek ve deneysel veriler ile karşılaştırmak için CSD den alınan kristal yap1 verileriyle GAUSSIAN 09 (Frisch vd., 2009) programında Yoğunluk Fonksiyonu teorisinin (DFT), B3LYP (Lee vd., 1988; Becke, 1993) ve HSE1PBE (Heyd ve Scuseria, 2004a,b; Heyd vd., 2005, 2006) metodunun 6-311++G(d,p) (Frisch vd., 1984) temel setinde hesaplanmıştır. Molekül 1a'nın optimize geometrinin yapısı Şekil 2'de verilmiştir.

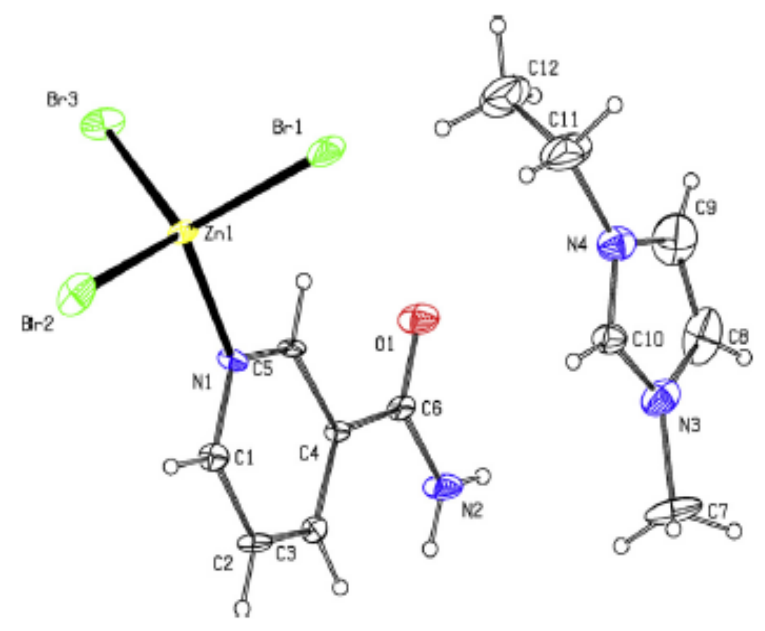

Şekil 1. Molekül 1a'nın atom numaraları

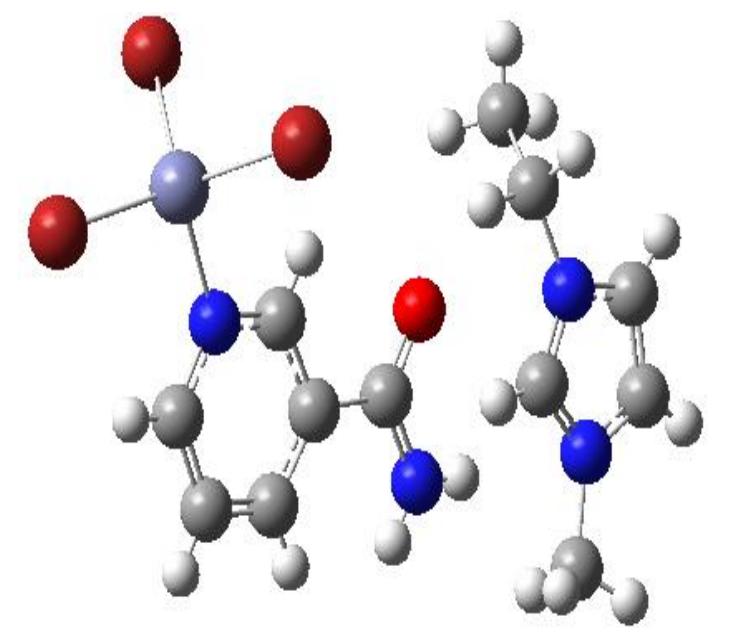

Şekil 2. HSE1PBE metodu kullanılarak molekül 1a'nın optimize geometrisi

Geometrik parametreler (bağ uzunluğu, bağ açısı) deneysel değerler ile birlikte Tablo 1'de listelenmiştir. Tablo 1'de deneysel ve teorik verilerimiz birbiri ile oldukça uyumlu olduğu görülmüştür.

\subsection{Kırmizl-alt ve Raman Spektrumu}

Molekül 1a için deneysel FT-IR spektrumu ile DFT medotuyla teorik olarak hesapladığımız kırmızı-altı (IR) spektrumu Şekil 3'de gösterilmiştir. Molekül 1a'nın $\mathrm{CH}$ gerilme bandı deneysel olarak $3065 \mathrm{~cm}^{-1}$ iken teorik olarak 3195 $\mathrm{cm}^{-1}$ ve $3015 \mathrm{~cm}^{-1}$ orta şidddette keskin olarak hesaplanmıştır. $\mathrm{C}=\mathrm{O}$ gerilme bandı ise deneysel olarak $1683 \mathrm{~cm}^{-1}$ iken teorik olarak $1755 \mathrm{~cm}^{-1}$ ve $1642 \mathrm{~cm}^{-1}$ güçlü ve keskin olarak hesaplanmıştır. Molekül 1a'nın deneysel Raman spektrumu ile DFT medotuyla teorik olarak hesapladığımız Raman spektrumu Şekil 4'de gösterilmiştir. 
Tablo 1. Deneysel (Chunyan vd., 2015) ve teorik olarak hesaplanan parametreler

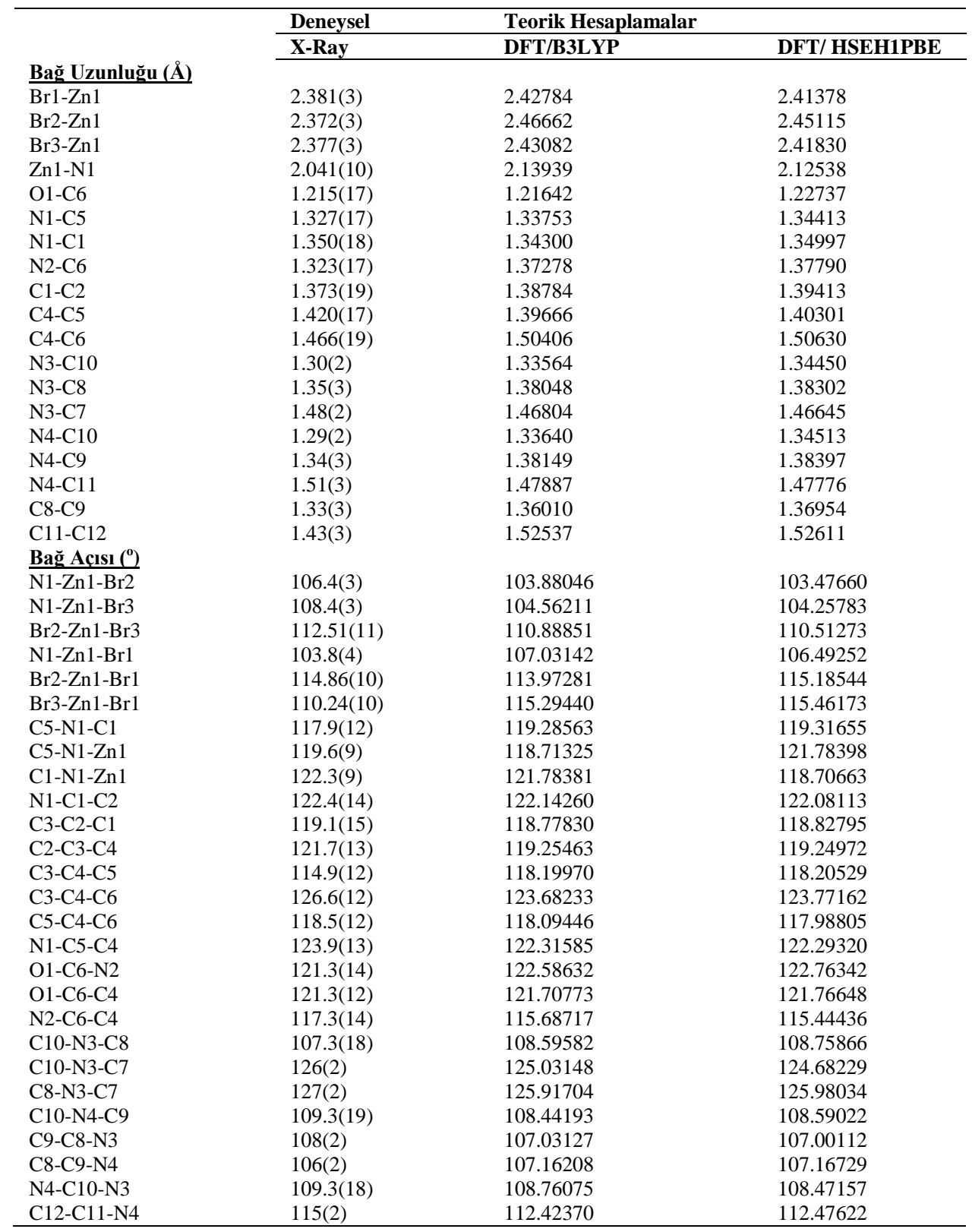

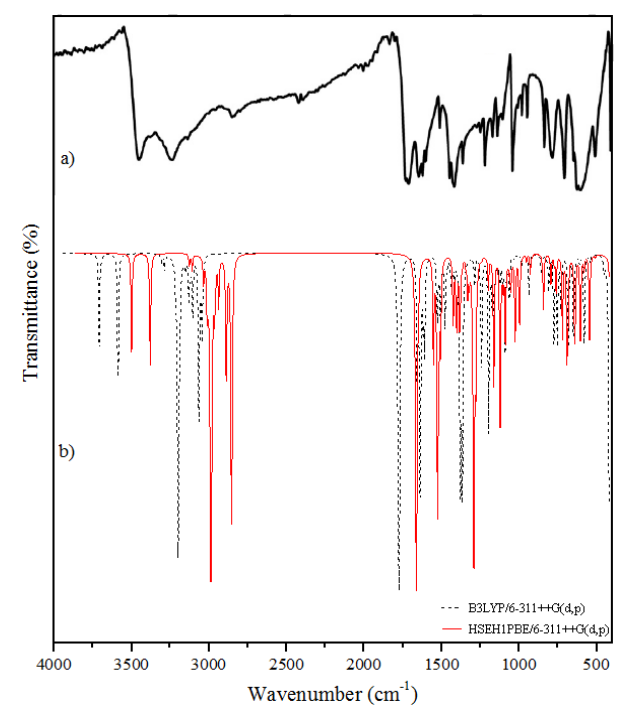

Şekil 3. (a) Deneysel (Chunyan vd., 2015) FT-IR (b) Teorik kırmızı-altı (IR) spektrumu

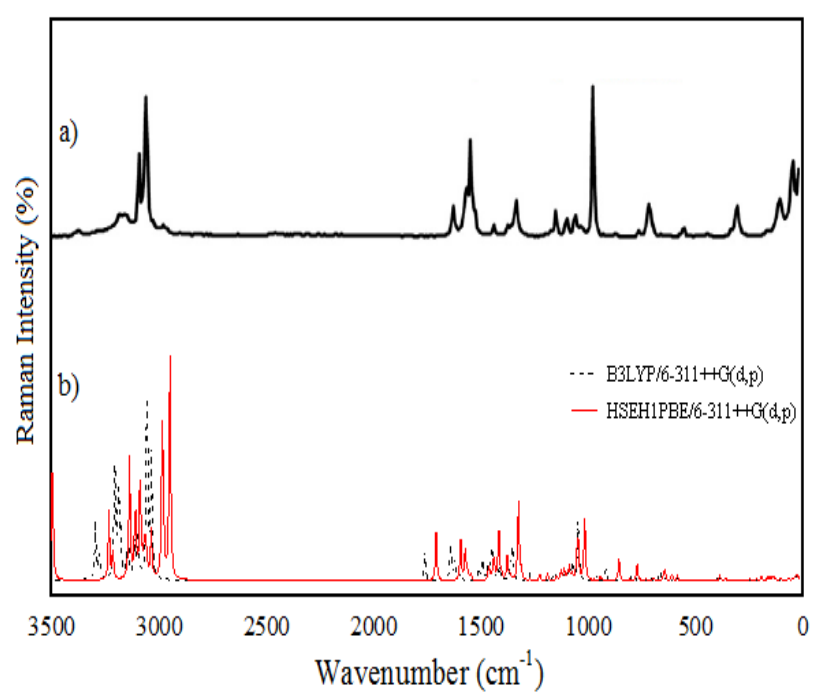

Şekil 4. (a) Deneysel (Chunyan vd., 2015) ve (b) Teorik Raman spektrumu 
Şekil 4'deki deneysel ve teorik Raman spektrumlarındaki titreşim bandları $3500-500 \mathrm{~cm}^{-1}$ arasındaki bölgede uyumlu, $500 \mathrm{~cm}^{-1}$ in altında ise band şiddetlerinde ise değişmeler gözlenmiştir.

Tablo 2'de verildiği gibi asimetrik $\mathrm{NH}_{2}$ gerilme titreşim bandı deneysel (Chunyan vd., 2015) 3404 $\mathrm{cm}^{-1}$ de çok güçlü olarak gözlenmişken B3LYP ve HSEH1PBE metotlarında hesaplanan teorik değerleri $3689 \mathrm{~cm}^{-1}$ ve $3480 \mathrm{~cm}^{-1}$ orta şiddette gözlemlenmiştir. Asimetrik $\mathrm{NH}_{2}$ gerilme titreşim band 1 ise deneysel (Chunyan vd., 2015) $3152 \mathrm{~cm}$ ${ }^{1} \mathrm{de}$ orta şiddette gözlenmişken B3LYP ve HSEH1PBE metotlarında hesaplanan teorik değerleri $3567 \mathrm{~cm}^{-1}$ ve $3360 \mathrm{~cm}^{-1}$ orta şiddette gözlemlenmiştir. Önemli piklerden olan CO gerilme titreşim bandı ise deneysel (Chunyan vd., 2015) $1683 \mathrm{~cm}^{-1}$ de çok güçlü olarak gözlenmişken B3LYP ve HSEH1PBE metotlarında hesaplanan teorik değerleri $1755 \mathrm{~cm}^{-1}$ ve $1641 \mathrm{~cm}^{-1}$ de çok güçlü şiddette gözlemlenmiştir.

Tablo 2. Deneysel (Chunyan vd., 2015) ve teorik titreşimsel dalgasayıları ve işaretlemeleri

\begin{tabular}{|c|c|c|c|c|c|c|}
\hline \multirow[b]{3}{*}{ Assigments } & \multicolumn{2}{|c|}{ Deneysel $\left(\mathrm{cm}^{-1}\right)$} & \multicolumn{4}{|c|}{ Teorik Hesaplamalar } \\
\hline & \multirow[b]{2}{*}{ IR } & \multirow[b]{2}{*}{ Raman } & \multicolumn{2}{|c|}{ B3LYP } & \multicolumn{2}{|c|}{ HSEH1PBE } \\
\hline & & & IR & Raman & IR & Raman \\
\hline$v_{\text {asym }}\left(\mathrm{NH}_{2}\right)$ & $3404 \mathrm{vs}$ & - & $3689.61 \mathrm{~m}$ & $3689.61 \mathrm{~m}$ & $3480.23 \mathrm{~m}$ & $3480.23 \mathrm{~s}$ \\
\hline$v_{\text {asym }}\left(\mathrm{NH}_{2}\right)$ & $3306 \mathrm{~m}$ & - & - & - & - & - \\
\hline$v_{\text {sym }}\left(\mathrm{NH}_{2}\right)$ & $3152 \mathrm{~m}$ & $3152 w$ & $3567.38 w$ & $3567.38 \mathrm{~s}$ & $3360.02 \mathrm{~m}$ & $3360.02 \mathrm{vs}$ \\
\hline$v_{\text {ring }}(\mathrm{CH})$ & $3065 \mathrm{~m}$ & $3069 \mathrm{~s}$ & $3195.78 \mathrm{vw}$ & 3195.78vs & $3015.89 w$ & $3015.89 v s$ \\
\hline$v_{\mathrm{im}}\left(\mathrm{CH}_{3}\right)$ & - & $2953 \mathrm{~m}$ & $3025.41 \mathrm{w}$ & $3025.41 \mathrm{vs}$ & $2863.65 \mathrm{w}$ & $2863.65 \mathrm{vs}$ \\
\hline$v(\mathrm{CO})$ & $1683 \mathrm{vs}$ & $1683 w$ & $1755.02 \mathrm{vs}$ & $1755.02 \mathrm{w}$ & 1641.96vs & $1641.96 \mathrm{~s}$ \\
\hline$\delta\left(\mathrm{NH}_{2}\right)$ & $1621 \mathrm{sh}$ & - & $1618.98 \mathrm{~s}$ & $1618.98 w$ & $1504.82 v s$ & $1504.82 \mathrm{~m}$ \\
\hline$v_{\mathrm{py}}($ ring $)$ & $1605 \mathrm{~s}$ & $1601 \mathrm{~s}$ & $1613.99 \mathrm{w}$ & $1613.99 w$ & $1532.09 \mathrm{~m}$ & $1532.09 \mathrm{~s}$ \\
\hline$v_{\mathrm{im}}(\mathrm{CN})$ & $1569 \mathrm{~s}$ & $1569 \mathrm{~m}$ & $1588.84 \mathrm{~m}$ & $1588.84 w$ & $1497.03 \mathrm{~m}$ & $1497.03 \mathrm{w}$ \\
\hline$v_{\mathrm{am}}(\mathrm{CN})$ & $1389 \mathrm{~m}$ & $1390 w$ & $1408.35 \mathrm{w}$ & $1408.35 \mathrm{~m}$ & $1359.88 \mathrm{~m}$ & $1359.88 \mathrm{~s}$ \\
\hline$v_{\mathrm{am}}(\mathrm{CN})$ & $1385 \mathrm{vs}$ & - & $1395.02 \mathrm{~m}$ & $1395.02 \mathrm{w}$ & $1323.22 \mathrm{w}$ & $1323.22 \mathrm{~m}$ \\
\hline$\delta_{\mathrm{py}}(\mathrm{CH})$ & $1329 w$ & $1199 w$ & $1358.68 \mathrm{~s}$ & $1358.68 \mathrm{~m}$ & $1272.24 \mathrm{vs}$ & $1272.24 \mathrm{~s}$ \\
\hline$v_{\text {im }}($ ring $)$ & $1169 \mathrm{~s}$ & $1165 \mathrm{~m}$ & $1176.13 \mathrm{~s}$ & $1176.13 \mathrm{vw}$ & $1101.65 \mathrm{~s}$ & $1101.65 \mathrm{~m}$ \\
\hline Ring def. $\delta_{\mathrm{py}}($ ring) & $1032 \mathrm{vs}$ & $1034 \mathrm{vs}$ & $1044.27 \mathrm{~m}$ & $1044.27 \mathrm{~s}$ & $1005.59 \mathrm{~m}$ & $1005.59 \mathrm{~m}$ \\
\hline $\mathrm{CH}$ op. Rock in imidazole ring & $757 \mathrm{~s}$ & $757 \mathrm{vw}$ & $733.23 \mathrm{~m}$ & $733.23 \mathrm{vw}$ & $671.46 \mathrm{~m}$ & $671.46 w$ \\
\hline Ring op. bend. $\gamma_{\mathrm{py}}$ (ring) & $691 \mathrm{vs}$ & $701 \mathrm{w}$ & $710.08 \mathrm{~m}$ & $710.08 \mathrm{vw}$ & $663.11 \mathrm{~m}$ & $663.11 w$ \\
\hline $\mathrm{NH}_{2}$ wagging & $646 s$ & $652 w$ & $661.69 \mathrm{~m}$ & $661.69 w$ & $616.73 \mathrm{~m}$ & $616.73 \mathrm{~m}$ \\
\hline Ring ip. def., $\delta_{\mathrm{py}}($ ring) & $621 \mathrm{~m}$ & - & $627.28 \mathrm{~m}$ & $627.28 w$ & $583.42 \mathrm{~m}$ & $583.42 w$ \\
\hline $\mathrm{CH}$ op. bend., $\gamma_{\mathrm{py}}(\mathrm{CH})$ & $516 \mathrm{~s}$ & - & $521.63 \mathrm{~m}$ & $521.63 \mathrm{w}$ & $488.73 \mathrm{vw}$ & $488.73 w$ \\
\hline$v(\mathrm{ZnN})$ & $424 \mathrm{~m}$ & $415 w$ & $431.81 \mathrm{~m}$ & $431.81 w$ & $397.88 w$ & $397.88 \mathrm{vw}$ \\
\hline$\delta(\mathrm{ZnBr})$ & - & $177 \mathrm{vs}$ & $190.46 \mathrm{~s}$ & $190.46 \mathrm{~m}$ & $185.03 \mathrm{~m}$ & $185.03 \mathrm{w}$ \\
\hline
\end{tabular}

\subsection{Elektronik Özellikleri}

Bir yapının elektron yoğunluğu dağılımına bak1larak, iyonizasyon potansiyeli, elektron ilgisi, kimyasal sertlik ve yumuşaklık parametreleri, elektrostatik potansiyel ve moleküler orbital şekilleri hakkında bilgi edinilebilir. Moleküler orbitaller HOMO-LUMO diye adlandırılır. Buradaki HOMO molekülün elektronu verme eğilimidir ve dolu olan en yüksek enerjili orbitaldir. LUMO ise molekülün elektron alma eğilimidir ve boş olan en düşük enerjili orbitaldir (Pir Gümüş ve Atalay, 2017). Molekülün enerji fark1 $(\Delta \mathrm{E})$ büyük olduğunda elektron dağılımı daha az değișime uğrar ve kutuplanma düşük olur. DFT/B3LYP ve DFT/HSE1PBE metotlarında 6$311++\mathrm{G}(\mathrm{d}, \mathrm{p})$ taban setinde elektronik yap1 parametreleri hesaplanmıştır ve Tablo 3'de bu parametreler listelenmiştir. Tablo 3'de verilen HOMO ile LUMO enerji farkının $1.5 \mathrm{eV}$ dan büyük olması termodinamik açıdan molekülün kararlı ve dayanıklı olduğunu gösterir. Ayrıca molekül kendi kendileri ile reaksiyon vermez, dimerleşme, polimerleşme gerçekleştirmez.

Tablo 3. Teorik elektronik yapı parametreleri

\begin{tabular}{lcc}
\hline & B3LYP & HSEH1PBE \\
\cline { 2 - 3 } $\mathrm{E}_{\text {HOMO }}(\mathrm{eV})$ & -6.08 & -5.18 \\
$\mathrm{E}_{\mathrm{LUMO}}(\mathrm{eV})$ & -2.08 & -2.80 \\
$\Delta \mathrm{E}(\mathrm{eV})$ & 3.99 & 2.39 \\
$\mathrm{I}(\mathrm{eV})$ & 6.08 & 5.18 \\
$\mathrm{~A}(\mathrm{eV})$ & 2.08 & 2.80 \\
$\chi(\mathrm{eV})$ & 4.08 & 3.99 \\
$\eta(\mathrm{eV})$ & 1.99 & 1.19 \\
$\left.\mathrm{~S}_{(\mathrm{eV}}{ }^{-1}\right)$ & 0.08 & 0.10 \\
$\mathrm{E}_{\text {TOTAL }}(\mathrm{a} . \mathrm{u})$ & -10263.96 & -10261.46 \\
\hline
\end{tabular}


HOMO ve LUMO dağılımlarının gösterimi şekil 5'de verilmiştir. Şekil 5'i incelediğimizde en düşük boş moleküler orbital olan LUMO enerjisi en fazla brom atomları üzerinde, en yüksek dolu moleküler orbital olan HOMO enerjisi ise piridin halkası üzerindeki bağlardadır.

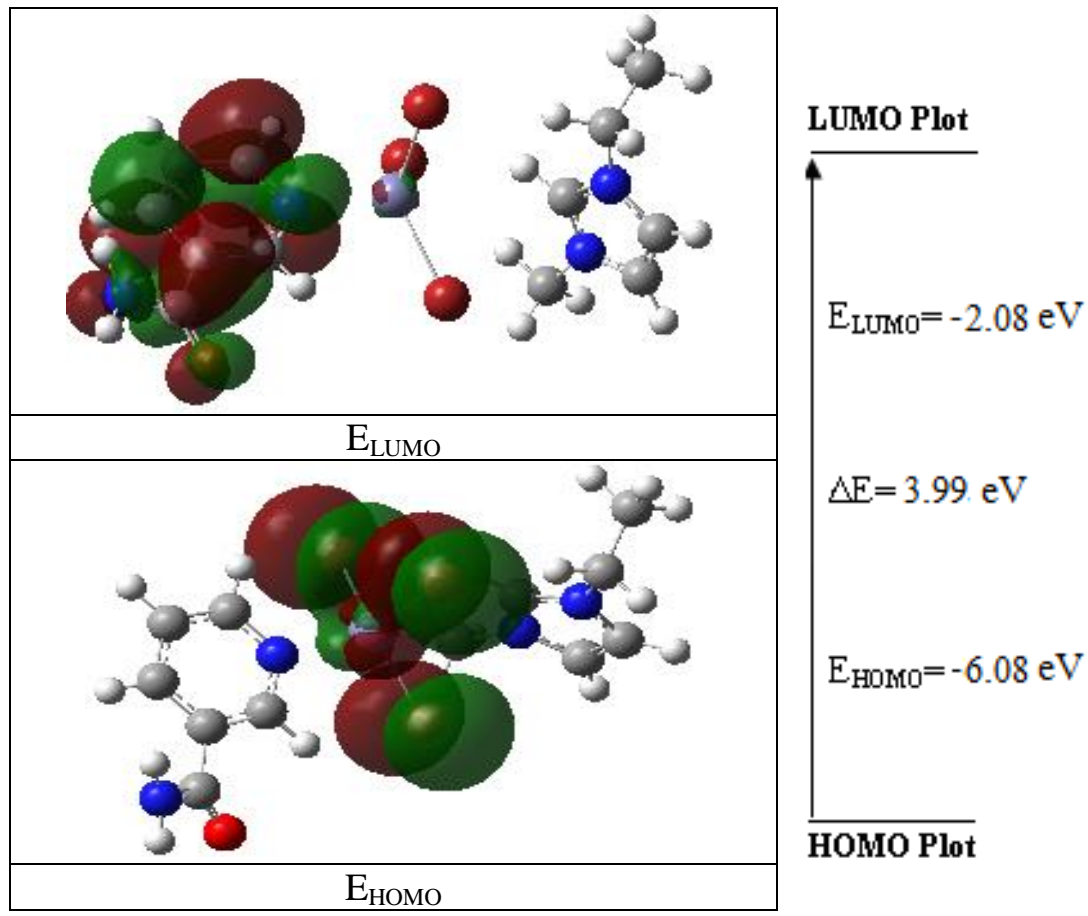

Şekil 5. Üç boyutlu HOMO ve LUMO'ların gösterimi

\subsection{Mulliken, APT ve NBO Yük Analizleri}

1a molekülünün moleküller arası etkileşimleri daha iyi anlayabilmek için moleküllerin atomik yükleri hesaplanıp Mulliken yoğunluk analizi, atomik polar tensör (APT) ve natural bağ orbital
(NBO) popülasyon analizi HSEH1PBE ve B3LYP metodlar1 ile hesaplanarak Tablo 4'de listelenmiștir. 1a molekülü için Mulliken, atomik polar tensör (APT) ve natural bağ orbital (NBO) yük analizleri daha iyi anlaşı1sın diye Şekil 6'da çizilmiştir.

Tablo 4. Teorik olarak hesaplanan Mulliken, APT ve NBO yük analizleri

\begin{tabular}{lllllll}
\hline Atom & Mulliken & & APT & & NBO & \\
\hline & B3LYP & HSEH1PBE & B3LYP & HSEH1PBE & B3LYP & HSEH1PBE \\
\hline C5 & -0.57797 & -0.80915 & 0.28346 & 0.26472 & 0.13598 & 0.11374 \\
C4 & 0.99026 & 1.18477 & -0.23649 & -0.20979 & -0.16294 & -0.15482 \\
C3 & -0.1027 & -0.12299 & 0.14733 & 0.11068 & -0.13651 & -0.15556 \\
C2 & -0.06705 & 0.01333 & -0.099 & -0.07475 & -0.22873 & -0.2309 \\
C1 & -0.03825 & -0.12513 & 0.26382 & 0.23611 & 0.10784 & 0.08615 \\
N1 & 0.34711 & 0.51805 & -0.52947 & -0.47335 & -0.53518 & -0.51441 \\
Zn1 & 0.69341 & 0.53706 & 1.36295 & 1.27267 & 0.77973 & 0.69563 \\
Br1 & -0.42773 & -0.35492 & -0.71534 & -0.68478 & -0.61706 & -0.58463 \\
Br2 & -0.46107 & -0.39138 & -0.77914 & -0.75159 & -0.62909 & -0.59417 \\
Br3 & -0.50452 & -0.44921 & -0.75379 & -0.72995 & -0.60805 & -0.57271 \\
C6 & -0.48665 & -0.67162 & 1.22258 & 1.132 & 0.65524 & 0.60429 \\
N2 & 0.18464 & 0.23261 & -0.30427 & -0.27305 & -0.80455 & -0.78939 \\
C9 & 0.15678 & 0.19572 & 0.1368 & 0.12606 & -0.01593 & -0.03074 \\
C8 & 0.02124 & 0.00118 & 0.136 & 0.12655 & -0.01702 & -0.03226 \\
C10 & -0.4014 & -0.69821 & 0.46768 & 0.44237 & 0.31382 & 0.27339 \\
N4 & 0.24561 & 0.41981 & -0.33453 & -0.28546 & -0.35742 & -0.33607 \\
C11 & 0.38946 & 0.43313 & 0.41726 & 0.38752 & -0.1882 & -0.22446 \\
N3 & 0.08596 & 0.21552 & -0.31308 & -0.27244 & -0.34842 & -0.32816 \\
C12 & -0.0174 & -0.07733 & 0.03132 & 0.03354 & -0.58163 & -0.61746 \\
C7 & 0.25941 & 0.19265 & 0.38577 & 0.35699 & -0.36274 & -0.40761 \\
O1 & -0.28913 & -0.24391 & -0.78986 & -0.73406 & -0.59197 & -0.55853 \\
\hline
\end{tabular}




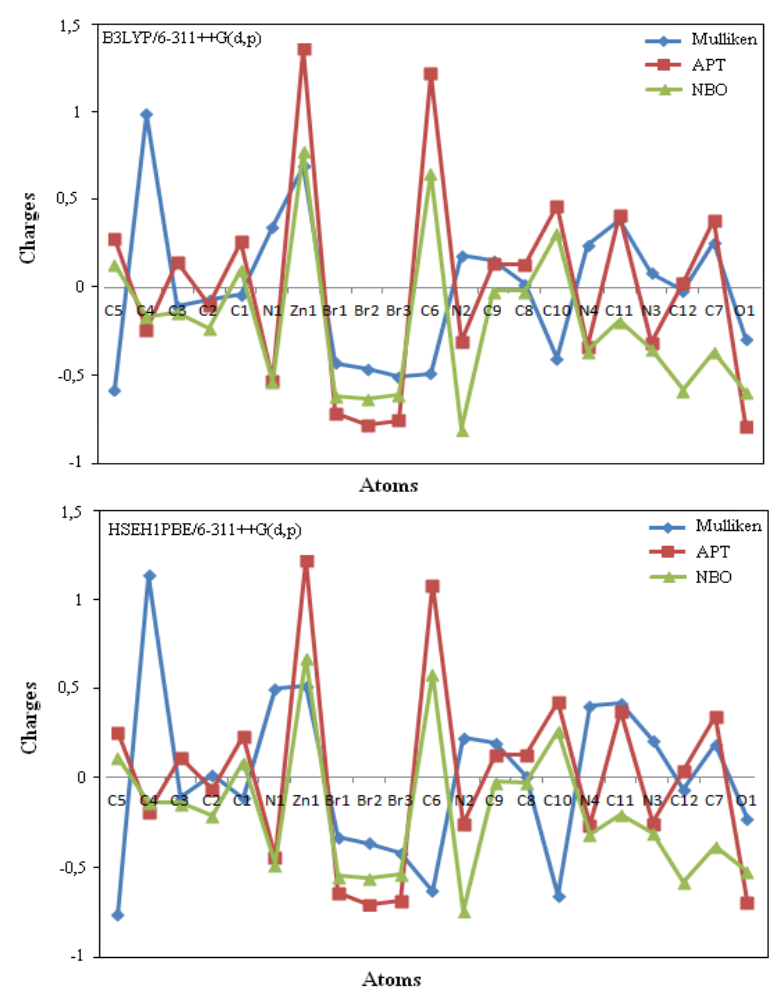

Şekil 6. Hesaplanan yük analizi

\subsection{Moleküler Elektrostatik Potansiyel Yüzeyi}

Moleküler elektrostatik potansiyel (MEP), moleküler davranışların reaktifliği, yapı aktifliği ve hidrojen bağlarını açıklamakta kullanılan faydalı bir metoddur. Kırmızı bölgeler (-) yüklü elektrostatik potansiyel bölgelerdir ve moleküllerin elektron yoğunluğu derişikliği tarafindan protonun çekimini (tek çiftlenim, $\pi$ bağları ...) temsil eder. Mavi bölgeler (+) yüklü elektrostatik potansiyel bölgelerdir ve düşük elektron yoğunluğunun ve nükleer yükün tam olarak korunmamış olduğu bölgelerde atom çekirdekleri tarafindan protonun itmesine karşılık gelir. Hesaplanan kısmi yükler ise sarı alanlar (-), kırmızı alanlar ise $(+)$ olmak üzere protonlar ve $(+)$ yüklere yaklaşılmasıyla molekülün nasıl bir etkileşim içinde olabileceğini gösterir.

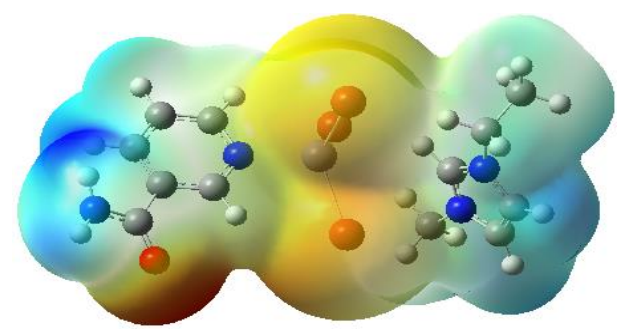

Şekil 7. Üç boyutlu moleküler elektronik potansiyel enerji yüzeyleri

1a molekülünün B3LYP/6-311G++(d,p) metodu ile optimize olan yapılarının 3 boyutlu moleküler elektrostatik potansiyel yüzey haritaları Şekil 7'de gösterilmiştir. Şekil 7 incelendiğinde, elektron yoğunluğunun en yüksek olduğu bölgenin oksijen $\mathrm{O} 1$ atomu civarında olduğu görülmektedir. Pozitif bölgeler ise, hidrojen atomları üzerinde mavi renk yoğunluğunun çoğunluklu olduğu bölgedir. N-H bölgesi de elektron yoğunluğunun en düşük olduğu bölgeleri göstermektedir.

\section{Sonuç ve Yorumlar}

Günümüzde kullanılan teorik hesaplama yöntemleri ile moleküllerin birçok özellikleri deneysel analizler yapılmasina gerek kalmadan incelenebilmektedir. Hatta bazı çalışmalarda deneysel yöntemden daha hassas ve güvenilir sonuçlar elde edilebilmektedir. Bir tek deneyle moleküler yapı, oluşum 1sıs1, dipol moment, iyonlaşma potansiyeli, elektron yükleri, elektron yoğunlukları, bağ uzunlukları gibi birçok bilgiyi verebilecek bir yöntem yoktur. Bu tür verilerin deneysel yöntem ile hesaplanmasında sonuçların güvenilirliği göz önünde bulundurulursa, yaygınlaşmakta olan birçok hesaplama yöntemi sonuçlarının, deneysel sonuçlara göre ne kadar güvenilir olduğu görülebilir.

$\mathrm{Bu}$ sebeple, 1a molekülünün teorik olarak kutuplanma etkisini (atomlar birbirine yaklaştırıldığında diğer çekirdeklerin etkisiyle elektronik yoğunluk bozulur) azaltmak gerekir. $\mathrm{Bu}$ sebeple eklenen polarize fonksiyonları ve uyarılmış, iyonik moleküllerde elektron yoğunluğunun molekülün temel durumuna göre daha dağınık olma durumunu modellemek için eklenen difüze fonksiyonları içeren 6$311++\mathrm{G}(\mathrm{d}, \mathrm{p})$ seviyesi ile incelenmiştir. Elde edilen minimum enerjili yapılar literatürde bulunan X-1şını yöntemi ile incelenen deneysel değerlerle karşılaştırılmıştır ve moleküllerin geometrik parametreleri deneysel değerlerle uyumlu olduğu bulunmuştur.

İlk olarak moleküllerin minimum enerjili yani kararlı yapısının bulunabilmesi amaciyla, geometri optimizasyonu yapılmıştır. İncelenen moleküllerin yapısı daha önceden X-1şını kırınım yöntemi ile belirlenmiş olduğundan, buradan elde edilen deneysel değerler, hesapladığımız geometrik parametrelerle karşılaştırılmıştır. Moleküllerin optimize yapısı kullanılarak harmonik olmayan titreşim frekansları hesaplanmıştır. Hesaplanan titreşim frekanslarının hangi titreşim türüne ait olduğunu belirleyebilmek için yani titreşimsel işaretlemelerin yapılması amaciyla benzer moleküller ve GaussView grafik ara yüzünde bulunan titreşim animasyonlarından yararlanılmıştır. Optimize edilen konfigürasyon- 
ların titreşim frekansları deneysel değerler ile uyumlu hale getirilebilmek için ölçekleme faktörleriyle çarpılıp düzeltilmiştir ve kırmızı-altı ile Raman spektrumları çizilmiştir. Deneysel değerler ile karşılaştırılarak iyi bir uyum içinde olduğu saptanmıştır. İncelenen 1a molekülünün öncü moleküler orbital enerjileri ve toplam enerjileri hesaplanmıştır. HOMO-LUMO enerji farklarına ve bu enerjilerden moleküler parametreler $(\eta ;$ moleküler sertlik ve $\chi$; elektronegatiflik) belirlenmiştir. Mulliken, APT ve NBO atomik yük değerlerinde ve NBO hibritleşme yüzdesi değişimleri incelendiğinde çok küçük değişimler olduğu görülmüştür. Öngörülen moleküllerin yapılarının optimize olmuş halinde izole moleküllere göre oluşan yük farklılıkları çalışmamızda moleküller arası etkileşimlerin delillerinden biri olarak ele alınmıştır. Ayrıca moleküler elektrostatik potansiyel enerji yüzey haritası (MEPS) belirlenmiştir.

Bu çalışmaların devamı olarak; daha büyük ve kompleks moleküler grupları içeren bileşiklerin daha kapsamlı bilgisayar sistemlerinde, yapılar üzerinde çalışmaların sürdürüleceği $\mathrm{NBO}$ ve konformasyon analizleri yapilabilir. Bilgisayarda hesaplanan simülasyonlar molekülün karakterizasyonunu belirlemekte yardımcı olur.

\section{Kaynaklar}

Akalin E. ve Akyuz S., 2006. Vibrational analysis of free and hydrogen bonded complexes of nicotinamide and picolinamide. Vibrational Spectroscopy, 42, 333-340.

Bakiler, M., Bolukbasi, O., ve Yilmaz, A., 2007. An experimental and theoretical study of vibrational spectra of picolinamide, nicotinamide, and isonicotinamide. Journal of Molecular Structure, 826, 6-16.

Becke, A. D., 1993. Density-functional thermochemistry. III. The role of exact exchange. Journal of Chemical Physics, 98, 5648 .

Bolukbasi, O., ve Akyuz, S., 2005. Computational vibrational study on coordinated nicotinamide, Journal of Molecular Structure, 744, 961-971.

Chunyan, L., Cui, F., Zhang, H. ve Xuan, X., 2015. Ionothermal synthesis, properties and vibrational spectra of zinc (II) complex with nicotinamide. Spectrochimica Acta A: Molecular and Biomolecular Spectroscopy, 134, 367-371.

Frisch M. J., Pople J.A., Binkley J.S. 1984. Selfconsistent molecular orbital methods 25 .
Supplementary functions for Gaussian basis sets. Journal of Chemical Physics, 80, 3265.

Frisch, M.J., Trucks, G.W., Schlegel, H.B., Scuseria, G.E., Robb, M.A., Cheeseman, J.R., Scalmani, G., Barone, V., Mennucci, B., Petersson, G.A., Nakatsuji, H., Caricato, M., Li, X., Hratchian, H.P., Izmaylov, A.F., Bloino, J., Zheng, G., Sonnenberg, J.L., Hada, M., Ehara, M., Toyota, K., Fukuda, R., Hasegawa, J., Ishida, M., Nakajima, T., Honda, Y., Kitao, O., Nakai, H., Vreven, T.,. Montgomery, J.A, Jr., Peralta, J.E., Ogliaro, F., Bearpark, M., Heyd, J.J., Brothers, E., Kudin, K.N., Staroverov, V.N., Kobayashi, R., Normand, J., Raghavachari, K., Rendell, A., Burant, J. C., Iyengar, S.S., Tomasi, J., Cossi, M., Rega, N., Millam, J. M., Klene, M., Knox, J.E., Cross, J. B., Bakken, V., Stratmann, R.E., Yazyev, O., Austin, A. J., Cammi, R., Pomelli, C., Ochterski, , V.G., Voth, G.A., Salvador, P., Dannenberg, J.J., Dapprich, S., Daniels, A.D., Farkas, O., Foresman, J. B., Ortiz, J.V., Cioslowski, J. ve Fox D.J., 2009. Gaussian 09, Revision A.1, Gaussian, Inc., Wallingford CT.

Heyd J. and Scuseria G.E. 2004b. Assessment and validation of a screened Coulomb hybrid density functional. Journal of Chemical Physics, 120,7274 .

Heyd J., Peralta J. E., Scuseria G. E., and Martin R. L. 2005. Energy band gaps and lattice parameters evaluated with the Heyd-Scuseria-Ernzerhof screened hybrid functional. Journal of Chemical Physics, 123, 174101.

Heyd J., Scuseria G. E., and Ernzerhof M. 2006. Hybrid functionals based on a screened Coulomb potential. Journal of Chemical Physics, 124, 219906.

Heyd, J. ve Scuseria G., 2004a. Efficient hybrid density functional calculations in solids: Assessment of the Heyd-Scuseria-Ernzerhof screened Coulomb hybrid functional. Journal of Chemical Physics, 121, 1187.

Ide, S., Ataç, A. ve Yurdakul, Ş., 2002. Spectroscopic and structural studies on dichlorobis (nicotinamide) zinc (II). Journal of Molecular Structure, 605, 103-107.

Lee, C., Yang, W. ve Parr R.G., 1988. Development of the Colle-Salvetti correlation-energy formula into a functional of the electron density. Physics Review B, 37, 785.

Magel, E., Hillinger, A.C., Wagner, T. ve Holl, W., 2001. Oxidative pentose phosphate pathway and pyridine nucleotides in relation to heartwood formation in Robinia pseudoacacia L. Phytochemistry 57, 1061-1068. 
Pasaoglu, H., Güven, S., Heren, Z. ve Büyükgüngör, O., 2006. Synthesis, spectroscopic and structural investigation of $\mathrm{ZnI} 2$ (nicotinamide) 2, ZnI 2 (isonicotinamide) 2 and $\left[\begin{array}{lllll}\mathrm{Zn} & (\mathrm{H} & 2 & \mathrm{O}\end{array}\right)$ (picolinamide) 2] I 2. Journal of Molecular Structure, 794, 270-276.

Pir Gümüş, H., Atalay, Y., 2017. 3-hidroksi-4hidroksimiinometil-5-hidroksimetil-1,2-

dimetilpiridinyum iyodid molekülünün geometrik yapısının incelenmesi, sakarya university journal of science, 21 (3), 564-571.

Raj, S.S., Fun, H.K., Zhao, P.S.,Jian, F.F., Lu, L.D., Yang, X.J. ve Wang, X., 2000. Bis (2-
aminopyridine-N) bis (benzoato-O) zinc. Acta Crystallographica C, 56, 742-743.

Shibata, K., 1994. Reparative Effect of Nicotinamide and Nicotinic Acid on the Aggravation of Tryptophan-nicotinamide Metabolism Caused by 6-Aminonicotinamide. Bioscience, Biotechnology Biochemistry, 58, 1729-1730.

Sismanoglu, T., 2003. Thermodynamics of stability constant of binary complex of nicotinamide with Mn. Chinese Chemical Letters, 14, 12071210. 